\title{
OPEN
}

Published online: 14 April 2020

\section{Publisher Correction: Inadequate Folic Acid Intake Among Women Taking Antiepileptic Drugs During Pregnancy in Japan: A Cross- Sectional Study}

\section{Yasuko Ikeda-Sakai, Yoshiyuki Saito, Taku Obara, Mikako Goto, Tami Sengoku, Yoshimitsu Takahashi $\mathbb{D}$, Hiromi Hamada, Takeo Nakayama \& Atsuko Murashima}

Correction to: Scientific Reports https://doi.org/10.1038/s41598-019-49782-x, published online 18 September 2019

The original version of this Article contained errors.

Affiliation 7 was a duplicate of Affiliation 5, and it has now been removed. The original Affiliation 8 is now listed as Affiliation 7.

In addition, in the Abstract,

"As planned pregnancy was the strongest factor, healthcare professionals should ensure that childbearing women taking antiepileptics are informed of the importance of planned pregnancy."

now reads:

"As unplanned pregnancy was the strongest factor, healthcare professionals should ensure that childbearing women taking antiepileptics are informed of the importance of planned pregnancy."

Moreover, in Table 1 row heading "Planned pregnancy, n (\%) Unplanned pregnancy" was incorrect.

"Planned pregnancy, n (\%) Unplanned pregnancy"

now reads:

"Planned pregnancy, n (\%)"

Finally, in the Discussion

"In the present study, a planned pregnancy was the strongest factor associated with folic acid intake"

now reads:

"In the present study, a planned pregnancy was the strongest factor associated with adequate folic acid intake" 


\begin{tabular}{|c|c|c|c|c|c|c|c|c|}
\hline \multirow[b]{3}{*}{ Age, mean (SD) } & \multirow{2}{*}{\multicolumn{2}{|c|}{ Total $(n=456)$}} & \multirow{2}{*}{\multicolumn{2}{|c|}{\begin{tabular}{|l|} 
Adequate intake \\
Before pregnancy \\
$(\mathrm{n}=76)$
\end{tabular}}} & \multicolumn{4}{|c|}{ Inadequate intake } \\
\hline & & & & & \multicolumn{2}{|c|}{$\begin{array}{l}\text { After pregnancy } \\
(\mathrm{n}=159)\end{array}$} & \multicolumn{2}{|c|}{$\begin{array}{l}\text { No intake } \\
(\mathrm{n}=221)\end{array}$} \\
\hline & 31.0 & $(5.4)$ & 32.8 & $(4.8)$ & 30.5 & $(4.6)$ & 30.7 & (5.8) \\
\hline \multicolumn{9}{|l|}{ Age (category), n (\%) } \\
\hline$<20$ years & 9 & $(2.0)$ & 0 & $(0)$ & 3 & $(1.9)$ & 6 & $(2.7)$ \\
\hline $21-29$ years & 165 & $(36.0)$ & 18 & $(23.4)$ & 66 & $(41.3)$ & 81 & $(36.7)$ \\
\hline $30-39$ years & 255 & $(56.1)$ & 51 & $(67.5)$ & 83 & $(52.5)$ & 121 & $(54.8)$ \\
\hline$\geq 40$ years & 27 & (5.9) & 7 & (9.1) & 7 & $(4.4)$ & 13 & (5.9) \\
\hline \multicolumn{9}{|l|}{ Body mass index, n (\%) } \\
\hline$<18.5$ & 86 & (18.9) & 15 & $(19.7)$ & 22 & $(13.8)$ & 49 & $(22.2)$ \\
\hline$\geq 18.5$ and $<25$ & 304 & $(66.7)$ & 53 & $(69.7)$ & 114 & $(71.7)$ & 137 & $(62.0)$ \\
\hline$\geq 25$ & 66 & $(14.5)$ & 8 & $(10.5)$ & 23 & $(14.5)$ & 35 & $(15.8)$ \\
\hline Smoking, n (\%) & 128 & $(28.1)$ & 9 & $(11.8)$ & 37 & $(23.3)$ & 82 & $(37.1)$ \\
\hline Alcohol drinking, n (\%) & 174 & $(38.2)$ & 17 & $(22.4)$ & 67 & $(42.1)$ & 90 & $(40.7)$ \\
\hline \multicolumn{9}{|l|}{ Parity, n (\%) } \\
\hline Primiparity & 357 & $(78.3)$ & 67 & $(88.2)$ & 121 & $(76.1)$ & 169 & $(76.5)$ \\
\hline Multiparity & 99 & $(21.7)$ & 9 & $(11.8)$ & 38 & $(23.9)$ & 52 & $(23.5)$ \\
\hline Spontaneous abortion, $\mathbf{n}(\%)$ & 72 & $(15.8)$ & 18 & $(23.7)$ & 18 & $(11.3)$ & 36 & $(16.3)$ \\
\hline Artificial abortion, $n$ (\%) & 100 & $(21.9)$ & 15 & $(19.7)$ & 31 & $(19.5)$ & 54 & $(24.4)$ \\
\hline Fertility treatment, $n(\%)$ & 26 & $(5.7)$ & 12 & $(15.8)$ & 12 & $(7.5)$ & 2 & $(0.9)$ \\
\hline Planned pregnancy, n (\%) & 160 & $(35.1)$ & 50 & $(65.8)$ & 55 & $(34.6)$ & 55 & $(24.9)$ \\
\hline \multicolumn{9}{|l|}{ Diseases under treatment, $\mathrm{n}(\%)$} \\
\hline Epilepsy & 178 & $(39.0)$ & 45 & $(59.2)$ & 73 & $(45.9)$ & 60 & $(27.1)$ \\
\hline Headache* & 18 & $(3.9)$ & 1 & $(1.3)$ & 10 & $(6.3)$ & 7 & $(3.2)$ \\
\hline Other neurological diseases $^{\dagger}$ & 18 & $(3.9)$ & 5 & (6.6) & 5 & $(3.1)$ & 8 & (3.6) \\
\hline Mental and behavioural disorders & 273 & $(59.9)$ & 35 & $(46.1)$ & 87 & $(54.7)$ & 151 & $(68.3)$ \\
\hline Collagen diseases & 6 & $(1.3)$ & 0 & $(0)$ & 2 & $(1.3)$ & 4 & $(1.8)$ \\
\hline Others & 80 & $(17.5)$ & 16 & $(21.1)$ & 22 & $(13.8)$ & 42 & $(19.0)$ \\
\hline
\end{tabular}

Table 1. Maternal characteristics. *Headache including migraine and cluster headache. ${ }^{\dagger}$ Other neurological diseases except epilepsy and headache.

Open Access This article is licensed under a Creative Commons Attribution 4.0 International License, which permits use, sharing, adaptation, distribution and reproduction in any medium or format, as long as you give appropriate credit to the original author(s) and the source, provide a link to the Creative Commons license, and indicate if changes were made. The images or other third party material in this article are included in the article's Creative Commons license, unless indicated otherwise in a credit line to the material. If material is not included in the article's Creative Commons license and your intended use is not permitted by statutory regulation or exceeds the permitted use, you will need to obtain permission directly from the copyright holder. To view a copy of this license, visit http://creativecommons.org/licenses/by/4.0/.

(C) The Author(s) 2020 